\title{
Findings from the Addressing Tobacco in Managed Care focus groups: an executive summary
}

\author{
Carol McPhillips-Tangum, Linda Schuessler
}

In 1997, the Robert Wood Johnson Foundation launched the four year Addressing Tobacco in Managed Care (ATMC) initiative designed to promote the implementation of evidence based tobacco interventions in managed care settings. As part of this initiative, a baseline survey of managed health care plans was conducted in 1997-98 for the primary purpose of assessing the extent to which health plans were developing, implementing, and evaluating evidence based tobacco cessation and prevention programs. A questionnaire was mailed to all health plans in the American Association of Health Plans' database of member and non-member plans and $323(60 \%)$ of health plans responded. The methods used to conduct the 1997-98 ATMC survey are reported more fully elsewhere. ${ }^{1}$ The results of the survey indicated, among other things, wide variation in the approaches taken by health plans to design and provide covered benefits for tobacco control programs. Owing to the high level of interest in identifying barriers to enhanced tobacco control programs, a qualitative approach (that is, focus groups) was adopted as a way to gain insight into the decision making process of health plans for these programs.

Highlights gleaned from the focus groups are presented here. A more detailed report is being prepared for future publication.

\section{Methods}

Data from the 1997-98 ATMC survey was used to categorise respondent health plans by the level and scope of tobacco related benefits and services provided to their members. For example, health plans were categorised as "maximum" providers of tobacco related benefits and services if they had established tobacco programs in place and provided coverage for pharmaceutical agents used to treat nicotine addiction. Health plans were categorised as "moderate" providers of tobacco related benefits and services if they had some programs in place but provided little or no coverage for tobacco related pharmaceuticals. Likewise, health plans were categorised as "minimum" providers if they had few, limited, or nascent tobacco related benefits or services in place. In order to have relatively homogenous focus groups, three separate focus groups were held to accommodate participants from the three categories of plans.

Participants for each focus group were identified from the information they provided on the 1997-98 ATMC survey. Names of potential participants were matched with names of health plan representatives registered to attend an upcoming ATMC conference. Invitations to participate in one of the three focus groups initially went out to representatives from health plans that had responded to the survey and registered to attend the conference. Remaining spaces $(n=15)$ in the focus groups were filled by inviting representatives from health plans who had not completed the survey, but were registered to attend the conference. When a representative from a health plan who did not respond to the survey was invited to participate in a focus group, data collected during or before recruitment was used to categorise the health plan as a maximum, moderate, or minimum provider of tobacco related programs and services. Ultimately, 10 health plan representatives participated in the maximum group, nine in the moderate group, and six in the minimum group.

The focus groups were held on 1 February 1999 during the second annual ATMC conference in San Diego, California. Each of the focus groups lasted approximately 90 minutes. Focus group participants were representatives from managed health care plans, and included health educators $(n=11)$, program administrators $(n=6)$, physicians $(n=5)$, quality improvement professionals $(n=3)$, and a researcher. A professional facilitator led each focus group using a protocol and topic guide developed in collaboration with researchers at the Prudential Center for Health Care Research. Each focus group was audiotaped and subsequently transcribed verbatim. The transcripts were then used to identify themes thought to provide insight into how health plans, at varying degrees of maturity and sophistication related to tobacco programs, make decisions about related benefits and services. A more detailed report is being prepared for publication and dissemination. However, this executive summary highlights some major themes.

\section{Results summary}

There were a number of interesting similarities and differences among participating health plans. It was apparent that nearly all offered a relatively wide assortment of tobacco control interventions and expressed a strong commitment to enhancing the services offered to health plan members. Low participation rates and the inability to identify smokers were important barriers identified for nearly all of the health plans represented in the focus groups. Frequently these two issues were linked, as many health plan representatives noted that their inability to identify individual smokers impeded the targeted outreach necessary for achieving optimal participation rates in ablueshieldca.com

C McPhillips-Tangum

L Schuessler 
cessation programs. Even participants representing the maximum group and often seen as "leaders in the field," voiced their frustration at still struggling to find solutions to these problems.

However, differences emerged among health plans in the manner in which decisions were made about tobacco related benefits and services. While participants from health plans in the moderate and maximum groups were easily able to describe how decisions about tobacco related benefits and services are made within their health plans, participants from the minimum group were unclear about how such decisions are (or should be) made in their respective health plans.

Differences also emerged among the three groups of health plans with respect to the perceived role of the purchaser. Participants in the minimum group seemed quite convinced that purchasers play a significant role in influencing which, if any, tobacco related services and benefits are offered in a particular health plan. Participants in the moderate group, on the other hand, believed that while purchasers play an important role in deciding which services will be covered, they do not necessarily play a role in determining which services will be offered. Interestingly, participants in the maximum group perceived the purchaser as one of many decision makers when it came to decisions about coverage issues, but they did not attribute any more authority to the purchaser than to other participants in the decision making process.

\section{Discussion}

The value of focus group research lies in deepening qualitative understanding of complex issues and helping to draw attention to areas deserving quantitative research. From the focus groups reported here, several themes emerged which bear further consideration.

The health plan's decision making process related to tobacco control was much clearer to participants in the moderate and maximum groups than in the minimum group. In the minimum group, the participants' lack of clarity about the decision making process may exist because questions about tobacco related benefits and services are not routinely raised, possibly because no one is designated to raise such questions. It appeared that health plans with persons who are designated the responsibility for addressing tobacco issues are more likely to do so. While this is not a surprising finding, it is an important one to confirm in larger and statistically sound samples of health plans. If confirmed, it suggests that health plans would be well served in their efforts to address tobacco by identifying a point person or "champion" to focus on this important topic, and it underscores the need for visible and influential senior leadership on this topic.

The respondents' perceptions of the role of the purchaser also provide insights. While many health plans in the minimum group indicated that purchasers played a major role in their decisions about tobacco services and benefits coverage, the purchaser's role was seen as less critical to health plans in the moderate and maximum groups. In considering the reasons for the differences in perception, it is possible that health plans in the moderate and maximum groups provide tobacco control interventions that are simply not influenced by economic or purchasing decisions. It is even more likely that the purchasers' role changes as a health plan "matures" with respect to its tobacco related services and benefits, and the purchaser becomes just one of a growing list of stakeholders. These interpretations suggest that active engagement of purchasers in discussions about the importance of tobacco control could serve as an inspiration for expansion of tobacco related benefits or services among health plans in the minimum group.

Finally, although some health plans are farther down the path than others in providing tobacco related benefits and services, nearly all participants voiced frustration as they struggle with low utilisation of these services and the inability to identify smokers, which would allow their recruitment into programs. As health plans search for answers to this dilemma, there is value to dissemination of information among health plans about effective strategies for identifying individual smokers and for increasing program participation. To satisfy this need, research should focus on identifying and evaluating strategies that hold the most promise.

Addressing issues such as those identified through the managed care focus groups will require a concerted effort across the industry. Health plans must continue to convene and collaborate, so that we can learn from one another's triumphs and disappointments. These findings also suggest priorities for the continued efforts of the ATMC program, including research, technical assistance, and future conferences and publications.

The ATMC program's National Technical Assistance Office (NTAO) provides highly valuable services for health plans and other organisations that are intent on finding effective and efficient approaches to reducing tobacco use. The NTAO disseminates cross cutting information and provides opportunities for sharing among organisations through a wide variety of channels, such as a quarterly newsletter, a listserv, regional workshops, and a national conference. These are described more fully elsewhere in this supplement. As more health plans become aware of and actively participate in this initiative, the benefits will continue to accrue for all involved.

The authors wish to thank the Robert Wood Johnson Foundation, the US Centers for Disease Control and Prevention, and the American Association of Health Plans for the funding necessary to conduct this research. The authors also acknowledge Julie to conduct this research. The authors also acknowledge Julie Brown for collaboration on the study protocol and facilitation of the focus groups, Maisha Kambon for assistance with cocus groups, and Tracy Orleans, Adele Franks, Carol Diamond, Mary Grace Flaherty, and Dianne Barker for reviewing and commenting on this manuscript. We also thank the participants of the focus groups for making this project possible.

1 McPhillips-Tangum C. Results from the first annual survey oddressing tobacco in managed care. Tobacco Control 1998; (suppl):S11-13. 